\title{
English Priming Effects on Spatial Decisions in Chinese
}

\author{
L. Suárez, A. B. B. Koh, and D. Zhang
}

\begin{abstract}
This study investigated the priming effects of English prepositions on spatial decisions in Chinese. Participants were English-Chinese bilinguals. One group of participants was exposed to written English sentences (primes) that contained the prepositions on, above, beneath, or below. Immediately after each sentence, an object was shown on the computer screen, and the participant was asked, in Chinese, to place a dot with the cursor either in a superior (上 shang) or inferior position (下 $x i a$ ) with respect to the object. Note that, in Chinese, shang includes on and above, and xia includes beneath and below. The results showed that when the participants were primed with on and beneath, they placed dots closer to the object than when they were primed with above and below. Another group of participants did the same experiment, but the priming sentences were written in Chinese. No priming effects were found. Results for the two experimental groups were compared and discussed.
\end{abstract}

Index Terms-Bilingualism, cognitive linguistics, language relativity, English, Chinese.

\section{INTRODUCTION}

Prepositions (e.g., on, above, beneath, below) indicate the location of objects in the space. The meaning of sentences comprising prepositions is achieved by judging geometric relationships between objects (e.g., in the sentence 'the bottle is above the glass', the preposition above indicates that the position of the bottle is higher in relation to the center of the glass) as well as considering extra geometrical relations between objects (i.e., evaluation of the function of the objects, and experience with objects interacting with each other in the space, determine how we infer their respective positions; e.g., in the sentence 'the bottle is above the glass', understanding is facilitated by imagining the bottle inclined towards the glass, probably pouring liquid) [1], [2].

Moreover, the use and comprehension of prepositions such as above and below seem to be more influenced by the position of the objects in relation to each other (i.e., geometry) than their functionality [2]. In contrast, other prepositions such as on seem to rely on functionality since on is typically employed with supporting objects (e.g., 'The book is on the shelf') [1].

In order to study the influence of geometry and function on the use of prepositions, Coventry et al. [2] employed several drawings in which objects fulfilled a function or not

Manuscript received April 4, 2013; revised May 12, 2013. L. Suárez thanks James Cook University (Singapore) for sponsoring the attendance and presentation of this work at ICSCH 2013 (Colombo, Sri Lanka, June 2013).

L. Suárez and A. B. B. Koh are with the James Cook University, 600 Upper Thomson Rd, 574421, Singapore (e-mail: lidia.suarez@jcu.edu.au, boonbei.koh@my.jcu.edu.au).

D. Zhang is with the National Institute of Education, 1 Nanyang Walk, 637616 Singapore (e-mail: dongbo.zhang@nie.edu.sg). depending on their position (e.g., a man holding a straight or tilted umbrella and not getting wet, or a man holding a straight or titled umbrella in such a way that he would get wet). Participants were shown these pictures and had to rate the appropriateness of sentences such as 'The man is below the umbrella' and 'The man is under the umbrella'. Coventry et al. found that below was more affected by the position of the umbrella with respect to the man (geometry) than under, which ratings were more affected by whether or not the umbrella protected the man from the rain (function). However, it is worth mentioning that Coventry et al. acknowledged that previous findings had found that below and under seem to be used similarly, and according to geometry, in experiments involving abstract figures with no real objects or plausible contexts.

Across languages, the use of geometry and function relationships for the same (or very similar) prepositions seems to be different. For example, Coventry and Guijarro-Fuentes [3] replicated Coventry et al.'s [2] study with Spanish prepositions and Spanish speakers. They found that function relationships drove the ratings of prepositions (over, under, below, and above) rather than geometry. Thus, different languages seem to employ different cues to comprehend the meaning of sentences containing prepositions.

Apart from Spanish, we believe that another language that could be deeply influenced by extra geometric variables is Chinese. In general, Chinese has extensive overlap of grammatical categories (e.g., the word gen can play different syntactic roles depending on its meaning: with, together, and, to follow, to go with), and Chinese words are more optional (vs. obligatory) than English words (i.e., it is syntactically permissible to omit most Chinese function words in a sentence without impairing its grammar or comprehensibility) [4]. Moreover, Chinese is a more homophonic and polysemous language than English inasmuch as different characters can be pronounced with the same pronunciation, and the same character may have different meanings depending on the context. Consequently, readers of Chinese rely heavily on context to figure out both meaning and pronunciation.

With regard to prepositions, English makes use of words such as on and above to indicate slightly differences between objects at superior positions. Geometrically, on is used to show that an object is above another object, they touch each other, and one of the objects is a surface (e.g., 'The plate is on the table'). However, according to the Cambridge dictionary (http://www.merriam-webster.com/dictionary/under) on can also have extra geometric meanings such as close proximity with (e.g., 'I stayed on her side'), source of support (e.g., 'To stand on one leg'), source of dependence (e.g., 'I depend on you'), means (e.g., 'Get on the bus', 'Talk on the phone'), 
possession of (e.g., 'She had a threat on her'), time (e.g., 'On Sunday'), manner of doing something (e.g., 'His work is on track'), status (e.g., 'To be on drugs'), participation (e.g., 'The band is on tour'), ' opposition (e.g., 'Put a gun on the hostage'), and so on. Above, on the other hand, refers to an object that is higher than another object (geometry) or higher in a rank or number. That is, on is a more polysemous word than above. Coventry et al. [2] discussed that prepositions that may be regarded as polysemous are more subjected to function reasoning than those prepositions that have one or a few meanings.

While English may use different words to refer to superior positions in the space, Chinese uses the preposition shang (上), which means in, up, before, earlier, over, on, on top of, and above, depending on the context. That is, the use of shang is very polysemous, for example, on (桌子上zhuozi shang / On the table), above (湖上 hu shang / Above the lake), in (历 史上 lishi shang / In the history), earlier (上星期 shang xingqi / Last week). Geometrically, shang can often be replaced by two other terms shang mian and shang fang, which are close in meaning to on and above, respectively. Without using its full forms of shang mian and shang fang, shang can be interpreted in English as either on or above, depending on their context of use. For example, 桌上有一个 杯子is best interpreted in English as 'on the table there is a cup'; 直升机在桥上盘旋 as “the helicopter is hovering above the bridge'. In both sentences, shang is used instead of respective use of shang mian and shang fang, which is not obligatory. Given the context, speakers of Chinese have no problem in resolving which meaning, shang mian and shang fang, shang refers to.

The same applies for prepositions indicating inferior positions such as beneath and below. In English, beneath means in a lower position, and usually suggests being in close contact or covered by something (e.g., 'The life jacket is beneath your seat'), and below refers to a lower position in a place, rank, or number. So, on and beneath both imply a very close position between objects, while above and below imply more distance between objects than on and beneath. In Chinese, the word xia (下) can be used to indicate beneath, below, under, and underneath. Like its counterpart shang, xia is also polysemous, for example, below (书下shu xia / Beneath the book), below (桥下 qiao xia / Below the bridge), off (台下 tai xia / Off the stage), later (下星期 xia xingqi / Next week). Geometrically, xia is often used instead of its two common full forms: xia mian and xia fang. Xia fang is more or less close to below, whereas xia mian can indicate beneath as well as below. Given the context, speakers of Chinese know whether xia refers to xia fang or xia mian, and whether xia mian means below or beneath. For example, the xia in书下有 只笔 ('Beneath the book there is a pen') can be replaced by its full form xia mian with the meaning of beneath, whereas the xia in 桥下有条船('Below the bridge there is a boat') can be replaced by both xia mian and xia fang, with the meaning of below, although xia mian seems to be more preferred. Like shang, the use of the full form xia mian and xia fang is very often not obligatory.

To sum up, English speakers employ prepositions that set apart slightly differences in distance between objects; Chinese speakers employ more general prepositions (shang, xia) even though phrasal forms of shang and xia are available that could distinguish distance differences. In addition, meanings in Chinese depend more on context than English.

In the current study, we explored English-Chinese bilinguals' spatial decisions when primed by English prepositions (e.g., There is a cup on the table), or Chinese prepositions (e.g., 桌子上有一个茶杯), embedded in sentences. For both conditions, the instructions in the subsequent spatial task were given in Chinese characters (e.g., 黑点在蓝物体上。请用鼠标点击出黑点的所在位置。 The black dot is in a superior position than the blue object. Please use the mouse cursor to indicate the location of the black dot). We expected that participants that read on and beneath in the priming sentence, would subsequently place the black dot closer to the blue object than when the same participants were exposed to prepositions such as above and below. In contrast, we expected not finding differences in distances when prime sentences were in Chinese, as the lexical / prepositional cue available in the Chinese primes, as opposed to the English ones, does not explicitly differentiate between on and above for shang, and beneath and below for xia.

\section{METHOD}

\section{A. Participants}

Seventy English-Chinese bilinguals, students of James Cook University, Singapore, participated for course credit or as volunteers. Participants' age ranged from 18 to 42 years old $(M=22.21)$. They had normal (or corrected-to-normal) visual acuity and no reading or any known developmental disorder.

\section{B. Design and Materials}

Participants were exposed to 96 trials, wherein there were 48 normal sentences (primes) and 48 nonsense sentences (primes), see examples in Table I. The meaning of the primes was the same in both the English-priming experiment and the Chinese-priming experiment. After each sentence, the participant decided whether the sentence made sense or not, and then worked on the spatial task, in which Chinese instructions requested the participant to put a black dot (with the cursor) in either a superior position (上 shang) or inferior position (下 xia) in relation to a blue object. See example in Fig. 1.

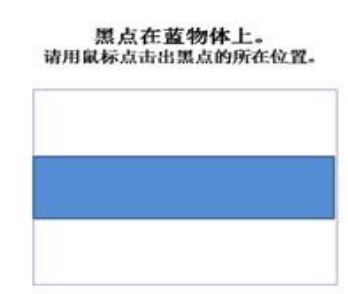

Fig. 1. Example of blue object used in the spatial decision task.

As shown in Table I, there were four possible conditions. To study priming effects, the statistical analyses were conducted only on trials that had normal sentences, and the same prepositions were used in the priming sentence and the spatial task. The analyzed trials are highlighted in bold in 
Table I (i.e., Normal sentence and Same preposition). The other conditions were fillers and were created to ensure that the priming sentences were being read (Normal vs. Nonsense sentence), and to disguise the objective of the experiment (e.g., using the preposition on as a prime and xia in the spatial task; i.e., different prepositions in the priming sentence and the spatial task).

TABLE I: EXPERIMENTAL GROUPS, AND TYPES AND EXAMPLES OF THE TRIALS EMPLOYED IN THE EXPERIMENT

\begin{tabular}{ll}
\hline \hline \multicolumn{1}{c}{ Prime } & \multicolumn{1}{c}{ Snglish-priming experiment } \\
\hline Normal sentence & Same preposition \\
The librarian places the & 黑点在蓝物体上 \\
reference book on the shelf & Different preposition \\
Normal sentence & 黑点在蓝物体下 \\
There is a cup on the table & Same preposition \\
Nonsense sentence & 黑点在蓝物体上 \\
Dogs live on clouds & Different preposition \\
Nonsense sentence & 黑点在蓝物体下 \\
The floor is on us & Shinese-priming experiment \\
\hline & Same preposition \\
Normal sentence & 黑点在蓝物体上 \\
图书馆理员把参考书放在架子 & \\
上 & Different preposition \\
Normal sentence & 黑点在蓝物体下 \\
桌子上有一个茶杯 & Same preposition \\
Nonsense sentence & 黑点在蓝物体上 \\
小狗住在云上 & Same preposition \\
Nonsense sentence & 黑点在蓝物体下 \\
地面在我们上 & \\
\hline \hline
\end{tabular}

In the spatial decision task, blue objects such as rectangles parallelograms, cylinders and so on, all of the same height, were inserted in the middle of a rectangle of $228 \times 181 \mathrm{~mm}$, which occupied most of a 350 X 270 LCD screen. The spatial instruction was presented above the rectangle, as in Fig. 1.

The design was 2 (Spatial task instruction: high position [上], low position [下]) X 2 (Prime: close [on, beneath], far [above, below]) X 2 (Language: English-priming, Chinese-priming). The first two variables were within-subjects measures, and the last one was a between-subjects measure.

The dependent variable was the average perpendicular distance between the participants' responses (i.e., wherein they placed the black dot) and the bottom horizontal line of the white rectangle, wherein the blue object was inserted. Note that the distance is not real distance, but distance between the coordinates. The values of the coordinates were registered by the E-prime software. The values were transformed to facilitate the interpretation of the distances (such as the bottom of the rectangle that contained the blue object was transformed to 0; see Fig. 2).

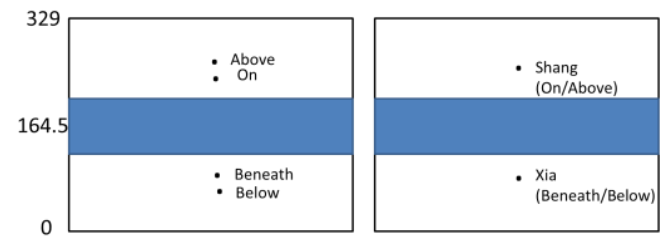

Fig. 2. English prepositions priming effects on spatial decisions in the English-priming experiment (left panel), and Chinese prepositions priming effects in the Chinese-priming experiment (right panel).

\section{Procedure}

The experiment was conducted in a computer lab equipped with E-prime. Participants in groups of four or less were randomly assigned to either the English-priming experiment or the Chinese-priming experiment. After completing a language background form, the participants performed the spatial decision task.

In the spatial decision task, each trial started with an instruction requesting the participant to click a small square in the middle of the screen. This was to ensure that the cursor was in the same position at the beginning of each trial. Immediately after clicking on the square, a sentence containing the preposition on, above, beneath or below (or shang and xia in the Chinese-priming condition) was shown on the screen, and participants had to decide whether the sentence made sense according to reality. If the sentence made sense, they pressed the left button of the mouse. If the sentence made no sense, they pressed the right button of the mouse. Immediately after the response, participants saw a blue object inserted in a rectangle (see Fig. 1), and on top of the figure was an instruction in Chinese requesting to place, with the cursor, a black dot in relation to the blue object. The placement was done once the participant clicked with the mouse. The different four types of trials were presented randomly.

Once the experiment was over, participants were asked whether or not they could guess the purpose of the study. Nobody guessed it.

The session lasted 30 minutes approximately.

\section{RESULTS}

A preliminary data analysis by items was performed to check that primes were read for comprehension. Priming effects could only be assumed if the participants were processing the intended prepositions. The average proportion of normal sentences responded accurately was .87 , and the average proportion of nonsense sentences responded accurately to was .90 . The difference reached significance, $F(1,6718)=16.48, M S E=.10, p<.001$, probably due to the large sample of items (96 items per participant, total 6720). The results, overall, indicated that participants were processing the prepositions.

In order to conduct the priming effects analyses, we selected trials in which the prime (e.g., on) and the instruction in the spatial decision task matched (i.e., shang). Moreover, we eliminated all trials that were responded wrongly. For example, an inaccurate trial is a trial wherein the Chinese instruction requested to place a dot on a superior position (shang), and the participant placed it below the middle line of the blue object that could divide the object into two equal parts (i.e., distance 164.5, see Fig. 2).

A 2 (Spatial task instruction: high position [上], low position [下]) X 2 (Prime: close [on, beneath], far [above, below]) X 2 (Language: English-priming, Chinese-priming) mixed ANOVA was performed. Two participants did not obtain data for some of the cells. Therefore, the analyses were run with 68 participants. Thirty-seven participants took part in 
the English-priming experiment and 31 participants in the Chinese-priming condition.

The analysis showed a main effect of Spatial task instruction, $F(1,66)=1407.26, M S E=1296.14, p<.001$. The analysis also showed a nonsignificant main effect of Language, $F<1$. Moreover, we found a significant interaction between Spatial task instruction and Prime, $F(1,66)=9.88$, $M S E=525.28, p=.003$, and a significant interaction between Spatial task instruction $\mathrm{X}$ Prime $\mathrm{X}$ Language, $F(1,66)=$ $11.48, M S E=525.28, p=.001$. Therefore, we proceeded to investigate the Spatial task instruction X Prime effects in each of the Language priming groups.

The results for the English-priming experiment and Chinese-priming experiment are depicted in Fig. 2 and Fig. 3.

Critically for our hypotheses, analyses revealed a Spatial task instruction $\mathrm{X}$ Prime interaction in the English-priming experiment, $F(1,66)=23.39, M S E=525.28, p<.001$. This suggested priming effects on the spatial task. Simple main effects of the significant interaction between Spatial task instruction and Prime, in the English-priming experiment, revealed that spatial decisions that had been primed with above $(M=260)$, were higher with respect to the blue object than spatial decisions for on $(M=242), F(1,36)=13.66$, $M S E=428.97, p=.001$. Also, spatial decisions for below $(\mathrm{M}$ =71) were lower than for beneath $(M=90), F(1,36)=12.71$, $M S E=505.94, p=.001$. These results supported our hypothesis regarding priming effects of English on Chinese spatial decisions.

The rest of the simple effects showed that responses to on $(M=242)$ and beneath $(M=90)$ differed in height, $F(1,36)=$ $1102.97, M S E=391.23, p<.001$, as well as above $(M=260)$ and below $(M=71), F(1,36)=429.00, M S E=1542.43, p$ $<.001$.

In contrast to the English-priming experiment, the results for the Chinese-priming experiment yielded a nonsignificant Spatial task instruction X Prime interaction, $F<1$, suggesting no priming effects. The only significant main effect in the Chinese-priming experiment was Spatial task instruction, wherein average distances for shang (average $M=244$ ) were higher than for xia (average $M=86$ ), $F(1,66)=596.62, M S E$ $=1296.14, p<.001$.

The direction of Spatial task instruction $\mathrm{X}$ Prime interaction for the English-priming experiment, and the lack of interaction between Spatial task instruction and Prime for the Chinese-priming experiment is shown in Fig. 3.

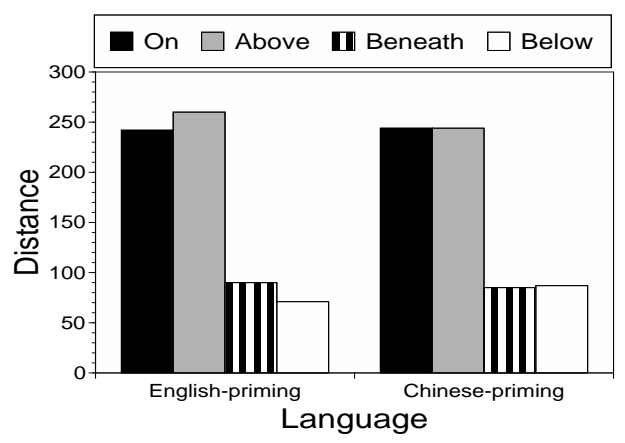

Fig. 3. Spatial responses due to priming effects of on, above, beneath, and below in the English-priming experiment (left panel), and spatial responses to the priming words shang (meaning on and above) and xia (meaning beneath and below) in the Chinese-priming experiment.

\section{DISCUSSION}

Languages constrain the way objects and situations are described. While English can be very specific by using propositions that indicate slightly different distances between objects (e.g., on and above), Chinese may use only one word (shang) to refer to both on and above. Chinese speakers rely heavily on the context and objects implicated in a sentence to imagine the distance between them. This study has shown that the concept of distance is not fixed but malleable, and easily affected not only by the context the objects are in, but also can be affected by the language being used.

Coventry et al. [2] mentioned that prepositions such as under and below are used similarly when there is no context attached to it, or the objects are abstract figures with no real functional relationship between them. In this study, we employed figures such as rectangles, and asked participants to place a dot either in a superior position (shang) or in an inferior position (xia) with regard to the object. That is, we used materials very different from Coventry et al.'s, who employed drawings of objects fulfilling a function (e.g., an umbrella used by a man to protect himself from the rain). Despite using geometrical figures in nonfunctional relationship contexts, our participants were influenced by the primes. In addition, the participants were not aware of the relationship between the primes and the spatial decision task. The results are interesting because priming effects for spatial decisions were obtained cross-linguistically and with nonfunctional objects.

The lack of significant difference between shang (meaning on) and shang (meaning above) and between xia (meaning beneath) and xia (meaning below) seems to have confirmed our hypothesis about the effect of Chinese prime sentences on Chinese-English bilinguals' spatial decision, given the lack of explicitness in distance encoding in shang and xia. However, considering that the participants had sufficient time to process the Chinese prime sentences with contextual clues, they should have appropriately interpreted those sentences with shang/on and shang/above sentences, and xia/beneath and xia/below. Consequently, there should have been some significant difference between shang/on and shang/above and between xia/beneath and xia/below. But this did not happen. One possible reason might be that some subjects relied for spatial decisions primarily on the Chinese instructions, which themselves included shang or xia and were the same for shang/on and shang/above primes (i.e., 黑点在蓝物体上 / The black dot is in a superior position than the blue object) and for xia/beneath and xia/below primes (i.e., 黑点在蓝物 体下 / The black dot is in an inferior position than the blue object). Given this possibility, future research might use nonverbal instructions for the spatial decision task or verbal instructions where shang or xia is not used.

Possible future questions to address are whether these results were due to a stronger priming effect for English than for Chinese primes due to the fact that most of the participants were more dominant in English than in Chinese. In relation to this issue, a study conducted by Altarriba and Canary [5], using the priming paradigm, showed stronger priming effects in English monolinguals than Spanish-English bilinguals. The experiment employed English primes and English targets to 
study the role of arousal during lexical access. Altarriba and Canary's results suggest that either the age of acquisition, the frequency a language is used, or both (and probably many other variables) can affect the priming effect. Therefore, it is likely that, in our study, priming effects were evidenced only when we used English words because this was the most dominant language of most of our students in Singapore. Samples with different proficiency in either English or Chinese will shed light on this query.

Another plausible explanation for the significant English-priming effects, and the lack of priming effects for Chinese, could be the fact that switching languages during the experiment produced more alertness and more attention towards the primes, resulting in larger priming effects.

An alternative explanation could be that the English primes above and below, which are used according to geometrical considerations [2], might have affected the spatial task, which was a geometrical task basically. In contrast, the Chinese prepositions are more polysemous and engage more in functional thinking. Consequently, thinking of such polysemous and functional words would not affect much the responses in the spatial decision task, which required the use of a geometrical dimension rather than an extra geometrical dimension.

Future research can also investigate whether spatial decisions prompted with nonverbal instructions are affected by prior thinking in either English or Chinese. Moreover, cross-linguistic research might research on whether different languages lead to estimate distances in different ways. More research is needed to understand how spatial distance and spatial decisions differ between monolinguals and bilinguals.

To conclude, this study has shown some of the effects of bilingualism on cognition, and the results indicated that thinking in one language (English) can affect spatial decisions prompted in Chinese.

\section{REFERENCES}

[1] K. R. Coventry, P. Guijarro-Fuentes, and B. Valdés, "Spatial language and second language acquisition, in V. Cook and B. Bassetti (Eds.),
Language and Bilingual Cognition, New York: Taylor \& Francis, 2011, pp. 263-286.

[2] K. R. Coventry, M. Prat-Sala, and L. Richards, "The interplay between geometry and function in the comprehension of 'over', 'under', 'above' and 'below'," J. Mem. \& Lang., vol. 112, pp. 202-213, 2001.

[3] K. R Coventry and P. Guijarro-Fuentes, "Las preposiciones en español y en inglés: la importancia relative del espacio y function," Cognitiva, vol. 16, pp. 73-93, 2004

[4] M. Palij and D. Aaronson, "The role of language background in cognitive processing," In R. J. Harris (Ed.), Cognitive Processing in Bilinguals, Amsterdam: Elsevier Science Publishers B. V., 1992, pp. 63-89.

[5] J. Altarriba and T. M. Canary, "The influence of emotional arousal on affective priming in monolingual and bilingual speakers," Journal of Multilingual and Multicultural Development, vol. 25, pp. 248-265, 2004.

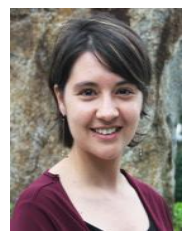

L. Suárez was born in Barcelona, Spain. She earned her $\mathrm{Ph} . \mathrm{D}$. in 2010 from the National University of Singapore. She currently works at James Cook University in Singapore as a lecturer and researcher. Her research interests include memory, bilingualism, linguistic relativity, and psycholinguistics. Dr. Suárez is member of the Language and Culture Research Center at the Cairns Institute, Australia; the American Psychology Society; and the Society for the Teaching of Psychology.

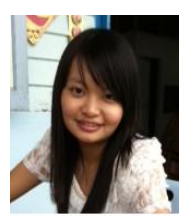

A. B. B. Koh was born in Johor Bahru, Malaysia. She gained her Bachelor of Psychology (Honours) in 2013 from the James Cook University Australia (Singapore Campus). She works at the Institute of Mental Health as a volunteer.

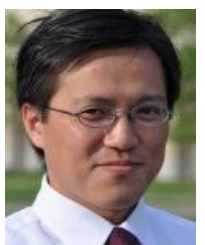

D. Zhang obtained his $\mathrm{PhD}$ degree in Carnegie Mellon University. $\mathrm{He}$ is a research scientist at the Centre for Research in Pedagogy \& Practice, National Institute of Education (NIE), Singapore. His research interests include second language acquisition, bilingualism and biliteracy, and applied psycholinguistics. With a grant from the Singapore Ministry of Education through NIE's Office of Education Research, he is currently investigating how bilingual children acquire reading skills concurrently in Singapore. 